\title{
COMPARISON OF HPAIV INFECTIVITY BETWEEN ADULTS AND JUVENILES DOMESTIC DUCKS AFTER NASAL INSTILLATION OF H5N6 VIRUS
}

\author{
PARK, J. E. - KIM, J. S. - HONG, Y. J. - SON, K. - KIL, J. - JHEONG, W. - KWON, J. T. \\ Biosafety Research Team, Environmental Health Research Department \\ National Institute of Environmental Research, Incheon 404-708, Republic of Korea \\ ${ }^{*}$ Corresponding author \\ e-mail:inhtox@gmail.com; phone:+82-32-560-7144; fax:+82-32-568-2038 \\ (Received 28 ${ }^{\text {th }}$ Mar 2019; accepted $13^{\text {th }}$ Jun 2019)
}

\begin{abstract}
Highly pathogenic avian influenza viruses (HPAIVs) can cause serious damage to wild birds and poultry. In this study, HPAIVs were administered via intranasal instillation to juvenile and adult domestic ducks, consisting of inoculation and contact groups, for comparative contagiousness. Oropharyngeal (OP) and cloacal (CL) swab analysis showed the overall virus shedding to be higher in OP than in CL. In addition, OP virus titer was higher in adults than in juveniles in the contact group. Our results indicate that adult susceptibility to AIV infection was greater than juvenile susceptibility, in case of samples from inoculated OP swab 2 days past inoculation (dpi) and contact OP swab 7 dpi. AIV distribution in the pancreas in juveniles was higher than that in adults; however, the virus was not detected in the trachea of either adults or juveniles. In conclusion, the results of this study suggest that differences in infectivity and prevalence of viruses in adults and juveniles may be influenced by the species of birds and neuraminidase enzymatic activity of the virus.
\end{abstract}

Keywords: highly pathogenic avian influenza virus, contact, inoculation, distribution, birds

\section{Introduction}

Avian Influenza virus (AIV), an influenza virus A of the family Orthomyxoviridae, is a single-stranded RNA virus with sixteen hemagglutinin (HA) subtypes (H1 to H16) and nine neuraminidase (NA) subtypes (N1 to N9) (Woo et al., 2017). Based on its pathogenicity, it is classified as either highly pathogenic AIV (HPAIV) or low pathogenic AIV (LPAIV). HPAIVs include $\mathrm{H} 5$ and $\mathrm{H}$, which are responsible for considerable illness or death in wild birds (Jeong et al., 2014).

In 1997, H5N1 HPAI outbreak occurred in chicken farms and live bird markets in Hong Kong, and the first case of chicken to human direct transmission of H5 was reported. Officially, H5N1 HPAIVs had emerged in 53 countries and human infections had occurred in 15 countries between 2003 and March 2014 (WHO).

In wildlife, the first HPAIV to cause the death of waterfowl was A/tern/South Africa/61 (H5N3) of H5 (Becker, 1966), following which H5N1 HPAIV appeared in many wild birds in Hong Kong (during 2002) and western China (Lake Qinghai; during 2005) (Ellis et al., 2004; Liu et al., 2005). Recently, HPAIVs have occurred throughout Asia, Europe, the Middle East, and several African countries linked to flyways (Olsen et al., 2006). Since wild birds, especially waterfowls, move their breeding site and wintering site, every winter, around 100,000-200,000 mallards arrive in Korea for spending the winter in various environments such as bird sanctuaries, inland small rivers, and lakes. In the spring, the waterfowls migrate for breeding; they pass through the east coast of North Korea after spanning North-eastern China (Kang et al., 2016). 
Since 2010, China has reported subtypes of Clade 2.3.4.4 H5 HPAIV in ducks (including H5N2, H5N5, H5N6, and H5N8) (Zhao et al., 2013; Su et al., 2015). The first report of Group C H5N6, clade 2.3.4.4, was from Korea and Japan, in fall 2016. After that, group C of H5N6 had been frequently detected in Korea during 2017 (Jeong et al., 2017; Woo et al., 2017). Despite reports of several types of H5 HPAIVs and comparison across the different subtypes, understanding of infection susceptibility across adults and juveniles is still lacking. Infection susceptibility is important to understand for the prevalence and management of diseases. Especially, comparison between adults and juveniles will be useful for understanding epidemiological consequences. In many wildlife diseases, juveniles have driven the spread of diseases (Ashby and Bruns, 2018). Therefore, in this study, we evaluated the susceptibility of H5N6 as a HPAIV (A/Whooper swan/Korea/Gangjin/W49-1/2016) at both adult and juvenile stages of ducks, and compared the infection sensitivity between the two.

\section{Materials and Methods}

\section{Experimental design}

The experimental groups consisted of 12 domestic ducks, each including a control group (Table 1). Animals confirmed negative for avian influenza virus infection in ELISA test (IDVet, Grabels, France, ID Screen Influenza A antibody Competition Multi-Species) prior to the experiment. Each control group $(n=4)$ was instilled with $0.2 \mathrm{ml}$ of phosphate-buffered saline (PBS) via the same exposure route. Five ducks were inoculated with H5N6 viruses via the choanal cleft using $0.2 \mathrm{ml}$ of $106.550 \%$ egg infectious dose (EID50)/100 $\mu \mathrm{l}$ in adult and juvenile, separately. After $8 \mathrm{~h}$, three healthy animals were cohoused with virus-injected animals for infectivity studies. All animal experiments were performed according to the Guidelines for Care and Use of the National Institute of Environmental Research (NIER 18-3) at biosafety level 3 (ABL3, KCDC-15-3-05) and were approved by Institutional Biosafety Committee (NIER-2018IBC-01).

Table 1. Group of animals used in the experiments

\begin{tabular}{l|c|c|c}
\hline \multirow{2}{*}{ Group } & \multicolumn{2}{c}{ Adult } & \multicolumn{2}{c}{ Juvenile } \\
\cline { 2 - 4 } & \multicolumn{2}{c}{ Number of Animals } \\
\hline \multirow{2}{*}{ Test } & Inoculation & 4 & 4 \\
\cline { 2 - 4 } & Control & 5 & 5 \\
\hline \multirow{2}{*}{ Contact } & 5 & 3 \\
\hline
\end{tabular}

Swab samples from the trachea and cloaca at days 1, 2, 3, 4, 5, 7, 10, and 14 were collected using transport tubes (Universal Viral Transport Standard Kit, BD). Samples were serially diluted 10-fold and titrated using specific pathogens free (SPF) eggs to determine EID50/0.1 ml. After 3 days post inoculation (dpi), two animals each of injection group and control group were euthanized, tissue and organ samples collected (such as trachea, lung, kidney, spleen, caecal tonsil, liver, intestine, pancreas, and proventriculus), and viral replication evaluated by real-time RT-PCR. RNA was isolated from $100 \mathrm{mg}$ of each tissue using a Maxwell RSC simpleRNA Tissue KIT (Promega, Madison) that was discovered using PowerCheck ${ }^{\mathrm{TM}}$ Influenza A Real-Time PCR Kit 
(Avian Influenza Virus Real-time PCR Kit, JSB-VT-023, JS Biotech, Korea) for nucleoprotein (NP) genes. Statistical analysis was performed using PRISM 5 software and two-way ANOVA (considering mean and standard deviation (SD)).

\section{Results and Discussion}

\section{Comparison of infectivity}

H5N6 virus from oropharyngeal (OP) and cloacal (CL) swab (A/Whooper swan/Korea/Gangjin/W49-1/2016/[H5N6]) of both adults and juveniles was isolated from 1-5 dpi and $7 \mathrm{dpi}$, and the overall viral shedding was confirmed to be higher in OP swab samples than in CL ones (Figure 1). In inoculated individuals, OP swab samples were also used to compare shedding between adults and juveniles. The first isolated virus at 2 dpi in adult samples showed the highest titer value (105.17 EID50/0.1 ml) of shedding. In addition, OP virus titer was higher in adults than in juveniles in the contact group (Figure 1). In inoculation and contact groups, results of OP swab samples at 2 dpi and 7 dpi were statistically significant $(P<0.01)$.
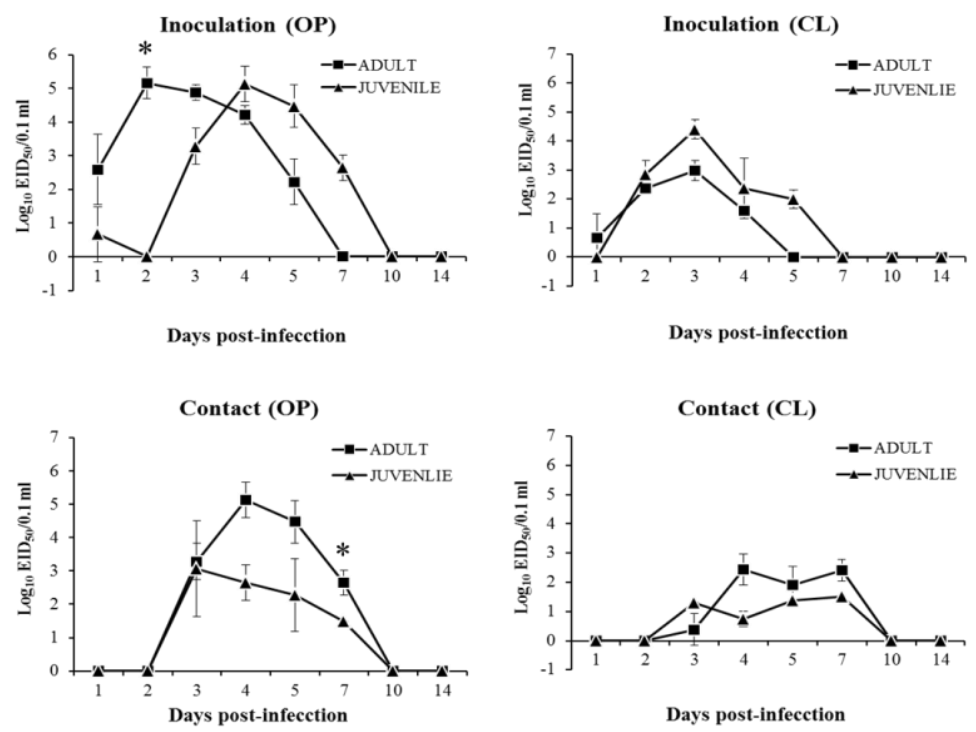

Figure 1. Comparison between adults and juveniles for the virus isolated from oropharyngeal

$(O P)$ and cloacal $(C L)$ swab of animals inoculated with 106.5 EID50/0.1 ml A/Whooper swan/Korea/Gangjin/W49-1/2016/[H5N6], and those non-inoculated but co-housed (Contact) with inoculated ones. Data were analyzed by two-way ANOVA with Bonferroni post-test, using

Prism 5 statistics software and calculating the mean and standard deviation $(S D)(n=3$, $* P<0.01)$

According to previous studies, immunity in juveniles is lower than that in adults, due to which, AIV infection is consistently higher in juveniles than in adults (Ip et al., 2008; Pybus et al., 2012). However, our results showed that adult susceptibility to AIV infection was greater than juvenile susceptibility, in case of samples from inoculated OP swab on 2 dpi and contact OP swab on 7 dpi (Figure 1). According to the seroprevalence study on wild waterfowl in Alaska, AIV antibodies in 11 species of wild waterfowls were higher in adults than in juveniles (Wilson et al., 2013), which was 
probably because the virus susceptibility depends on the exposure of AIV to the species. Same pattern had also been shown in pink-footed geese (Anser brachyrhynchus) and Bewick's swans (Cygnus columbiannus bewickii) (Hoye et al., 2011a, b).

Moreover, reproduction might also affect influenza host susceptibility (van Dijk et al., 2018). During breeding season, most animals, including birds, use lots of energy in reproduction, hence showing relatively lower immunity than in non-breeding season. Adult individuals in our study were 22-week-old, and since ducks generally breed during May-July, their testicles were found enlarged upon autopsy (Blums et al., 2002; Ashby and Bruns, 2018). Therefore, we suspect that reproduction activity promotes higher infection susceptibility in adult than in juveniles.

The tissue from animals occurred in most of the internal organs of both adult and juvenile from H5N6, except for the trachea, caecal tonsil, intestine, proventriculus, brain, and muscle. AIV distribution in the pancreas from juveniles was higher than that from adults; however, the virus was not detected in the trachea from either adults or juveniles (Table 2). Although trachea is one of the main organs for detecting AI virus, almost no virus was detected in our study, which might be explained based on the report by Das et al. (2008), which showed that tracheal swab is less sensitive for detecting AIV in preclinical chickens.

Table 2. AIV distribution in tissues of infected duck (both adult and juvenile)

\begin{tabular}{c|c|c|c|c|c|c|c|c|c|c|c}
\hline Age & Tra $^{\text {a }}$ & Lung & Kid & Spl & CT & Liv & Int & Pan & Pro & Brain & Mus \\
\hline Adult1 & $-{ }^{\mathrm{b}}$ & + & ++ & ++ & - & + & - & ++ & - & + & - \\
\hline Adult2 & - & - & - & - & - & + & - & ++ & - & - & - \\
\hline Juvenile1 & - & + & ++ & - & - & - & - & +++ & - & - & - \\
\hline Juvenile2 & + & ++ & +++ & ++ & - & + & + & +++ & - & + & - \\
\hline $\begin{array}{c}\text { Control } \\
\text { (PBS) }\end{array}$ & - & - & - & - & - & - & - & - & - & - & - \\
\hline
\end{tabular}

aTra; Trachea, Kid; Kidney, Spl; Spleen, CT; Caecal Tonsil, Liv; Liver, Int; Intestine, Pan; Pancreas, Pro; Proventriculus, Mus; Muscle

${ }^{\mathrm{b}_{+++}} ; 20<\mathrm{CT}<25,++; 25<\mathrm{CT}<30,+; 30<\mathrm{CT}<35,-; \mathrm{CT}<35$

Mucin secretion is an important factor for the prevention of influenza virus infection in the airway (Burnet, 1948; Zanin et al., 2016). Especially, between influenza virus NA enzyme activity and mucus barrier may be affection in infectivity (Cohen et al., 2013; Zanin et al., 2015). Previous study had reported no H5N6 virus infectivity due to aerosol diffusion in ferret model (Sun et al., 2016). In the present study, our results demonstrated that the H5N6 virus is spread by direct contact between individuals and that the infectivity depends on the NA enzyme activity in duck model.

\section{Conclusion}

Our results suggest that differences in infectivity and prevalence of virus in adults and juveniles may be influenced by the species of birds, breeding season and NA enzymatic activity of the virus. This information may be helpful in further study to understand age-dependent infection sensitivity of avian species and affection of reproduction and these reasons should be considering when monitoring AIV. 
Acknowledgements. This work was supported by a grant from the National Institute of Environment Research (NIER), funded by the Ministry of Environment (MOE) of the Republic of Korea (NIERRP2018-114).

\section{REFERENCES}

[1] Ashby, B., Bruns, E. (2018): The evolution of juvenile susceptibility to infectious disease. - Proc. R. Soc. B 285(1881): 20180844.

[2] Becker, W. B. (1966): The isolation and classification of tern virus: Influenza Virus A/Tern/South Africa/1961. - The Journal of Hygiene 64(3): 309-320.

[3] Blums, P., Nichols, J. D., Hines, J. E., Mednis, A. (2002): Sources of variation in survival and breeding site fidelity in three species of European ducks. - Journal of Animal Ecology 71(3): 438-450.

[4] Burnet, F. M. (1948): Mucins and mucoids in relation to influenza virus action: v. The destruction of "francis inhibitor" activity in a purified mucoid by virus action. Australian Journal of Experimental Biology and Medical Science 26(5): 389-402.

[5] Cohen, M., Zhang, X.-Q., Senaati, H. P., Chen, H.-W., Varki, N. M., Schooley, R. T., Gagneux, P. (2013): Influenza A penetrates host mucus by cleaving sialic acids with neuraminidase. - Virology journal 10(1): 321.

[6] Das, A., Spackman, E., Thomas, C., Swayne, D. E., Suarez, D. L. (2008): Detection of H5N1 high-pathogenicity avian influenza virus in meat and tracheal samples from experimentally infected chickens. - Avian diseases 52(1): 40-48.

[7] Ellis, T. M., Barry Bousfield, R., Bissett, L. A., Dyrting, K. C., Luk, G. S., Tsim, S., Sturm-Ramirez, K., Webster, R. G., Guan, Y., Peiris, J. M. (2004): Investigation of outbreaks of highly pathogenic H5N1 avian influenza in waterfowl and wild birds in Hong Kong in late 2002. - Avian Pathology 33(5): 492-505.

[8] Hoye, B. J., Fouchier, R. A., Klaassen, M. (2011a): Host behaviour and physiology underpin individual variation in avian influenza virus infection in migratory Bewick's swans. - Proceedings of the Royal Society B: Biological Sciences 279(1728): 529-534.

[9] Hoye, B. J., Munster, V. J., Nishiura, H., Fouchier, R. A., Madsen, J., Klaassen, M. (2011b): Reconstructing an annual cycle of interaction: natural infection and antibody dynamics to avian influenza along a migratory flyway. - Oikos 120(5): 748-755.

[10] Ip, H. S., Flint, P. L., Franson, J. C., Dusek, R. J., Derksen, D. V., Gill, R. E., Ely, C. R., Pearce, J. M., Lanctot, R. B., Matsuoka, S. M. (2008): Prevalence of influenza A viruses in wild migratory birds in Alaska: patterns of variation in detection at a crossroads of intercontinental flyways. - Virology journal 5(1): 71.

[11] Jeong, J., Kang, H.-M., Lee, E.-K., Song, B.-M., Kwon, Y.-K., Kim, H.-R., Choi, K.-S., Kim, J.-Y., Lee, H.-J., Moon, O.-K. (2014): Highly pathogenic avian influenza virus (H5N8) in domestic poultry and its relationship with migratory birds in South Korea during 2014. - Veterinary microbiology 173(3-4): 249-257.

[12] Jeong, J., Woo, C., Ip, H. S., An, I., Kim, Y., Lee, K., Jo, S.-D., Son, K., Lee, S., Oem, J.K. (2017): Identification of Two novel reassortant avian influenza a (H5N6) viruses in whooper swans in Korea, 2016. - Virology journal 14(1): 60.

[13] Kang, T., Kang, Y.-M., Jeong, W., Moon, O.-K., Yoon, H., Choi, J., Lee, H. (2016): Spring migration of mallards (Anas platyrhynchos) tracked with wild-trackers in East Asia. - Journal of Asia-Pacific Biodiversity 9(3): 323-327.

[14] Liu, J., Xiao, H., Lei, F., Zhu, Q., Qin, K., Zhang, X.-W., Zhang, X.-1., Zhao, D., Wang, G., Feng, Y. (2005): Highly pathogenic H5N1 influenza virus infection in migratory birds. - Science 309(5738): 1206-1206.

[15] Olsen, B., Munster, V. J., Wallensten, A., Waldenström, J., Osterhaus, A. D., Fouchier, R. A. (2006): Global patterns of influenza A virus in wild birds. - Science 312(5772): 384388. 
[16] Pybus, O., Perrins, C., Choudhury, B., Manvell, R., Nunez, A., Schulenburg, B., Sheldon, B., Brown, I. (2012): The ecology and age structure of a highly pathogenic avian influenza virus outbreak in wild mute swans. - Parasitology 139(14): 1914-1923.

[17] Su, S., Bi, Y., Wong, G., Gray, G. C., Gao, G. F., Li, S. (2015): Epidemiology, evolution, and recent outbreaks of avian influenza virus in China. - Journal of virology 89(17): 8671-8676.

[18] Sun, H., Pu, J., Wei, Y., Sun, Y., Hu, J., Liu, L., Xu, G., Gao, W., Li, C., Zhang, X. (2016): Highly pathogenic avian influenza H5N6 viruses exhibit enhanced affinity for human type sialic acid receptor and in-contact transmission in model ferrets. - Journal of virology: JVI. 00127-16.

[19] van Dijk, J. G., Verhagen, J. H., Wille, M., Waldenström, J. (2018): Host and virus ecology as determinants of influenza A virus transmission in wild birds. - Current opinion in virology 28: 26-36.

[20] WHO: Available online: https://www.who.int/influenza/human_animal_interface/en/.

[21] Wilson, H. M., Hall, J. S., Flint, P. L., Franson, J. C., Ely, C. R., Schmutz, J. A., Samuel, M. D. (2013): High seroprevalence of antibodies to avian influenza viruses among wild waterfowl in Alaska: implications for surveillance. - PLoS One 8(3): e58308.

[22] Woo, C., Kwon, J.-H., Lee, D.-H., Kim, Y., Lee, K., Jo, S.-D., Dong Son, K., Oem, J.-K., Wang, S.-J., Kim, Y. (2017): Novel reassortant clade 2.3. 4.4 avian influenza A (H5N8) virus in a grey heron in South Korea in 2017. - Archives of virology 162(12): 3887-3891.

[23] Zanin, M., Marathe, B., Wong, S.-S., Yoon, S.-W., Collin, E., Oshansky, C., Jones, J., Hause, B., Webby, R. (2015): Pandemic swine H1N1 influenza viruses with almost undetectable neuraminidase activity do not transmit via aerosols in ferrets and are inhibited by human mucus, but not swine mucus. - Journal of virology: JVI. 02537-14.

[24] Zanin, M., Baviskar, P., Webster, R., Webby, R. (2016): The interaction between respiratory pathogens and mucus. - Cell host \& microbe 19(2): 159-168.

[25] Zhao, K., Gu, M., Zhong, L., Duan, Z., Zhang, Y., Zhu, Y., Zhao, G., Zhao, M., Chen, Z., $\mathrm{Hu}$, S. (2013): Characterization of three H5N5 and one H5N8 highly pathogenic avian influenza viruses in China. - Veterinary microbiology 163(3-4): 351-357. 